\title{
New anticoagulant drugs versus warfarin in atrial fibrillation: economic evaluation and cost-effectiveness analysis
}

\author{
Mauro Silingardi \\ Unità Internistica Multidisciplinare, Guastalla, AUSL, Reggio Emilia, Italy
}

\begin{abstract}
Health care resources available for medical procedures, including pharmaceuticals, are limited worldwide. Health economic evidence is now accepted as an essential component of health technology appraisal, realizing the importance of value for money considerations for a more efficient (cost-effective) prescribing. Regulatory agencies in more and more countries perform economic evaluation and cost-effectiveness analysis in order to decide about reimbursement of a new and almost always more expensive drug. Pharmacoeconomy is now acknowledged as a science. Cost-effective analysis is just one of its approaches, measuring cost in money and benefit in terms of Quality Adjusted Life Year, a new outcome measure which combines quantity/quality of additional life-years gained with the new drug/technology. A growing body of pharmacoeconomic evidence about new anticoagulant drugs (dabigatran, rivaroxaban, apixaban) for stroke prevention in atrial fibrillation is now available. Most of this evidence comes from the National Institute of Health and Clinical Excellence (NICE) in the United Kingdom, the most referenced regulatory agency in the world. Compared to current standard therapies (warfarin), dabigatran, rivaroxaban and apixaban are cost-effective treatments for the whole population of patients with atrial fibrillation, independently of poor/good international normalized ratio control (time in therapeutic range) and risk stratification for stroke (CHADS2 score). Significant innovation and the lower rate of intracranial hemorrhage/hemorrhagic stroke coupled with the new drugs are the key drivers of these results.
\end{abstract}

\section{Introduction}

Worldwide, warfarin is one of the most prescribed drugs. The main indication for oral anticoagulant therapy is atrial fibrillation (AF). Given the high prevalence of $\mathrm{AF}$ in older individuals and the aging population an ever-increasing number of people are expected to start warfarin. The drawbacks in its use are well known and are well reviewed elsewhere in this journal. ${ }^{1}$ Dabigatran, rivaroxaban and apixaban are new anticoagulant drugs with the potential of re-

Correspondence: Mauro Silingardi, Unità Internistica Multidisciplinare, Guastalla, AUSL, Reggio Emilia, Italy. E-mail: mauro.silingardi@ausl.re.it

Key words: cost effectiveness, economic evaluation, dabigatran, rivaroxaban, apixaban, warfarin, stroke prevention, atrial fibrillation.

Received for publication: 11 April 2013.

Revision received: Not required.

Accepted for publication: 16 May 2013.

This work is licensed under a Creative Commons Attribution NonCommercial 3.0 License (CC BY-NC 3.0).

CCopyright M. Silingardi, 2013

Licensee PAGEPress, Italy

Italian Journal of Medicine 2013; 7(s8):65-70

doi:10.4081/itjm.2013.s8.65 placing oral anticoagulant therapy, as clearly demonstrated by several randomized controlled trials (RCT).

Substituting a new anticoagulant for warfarin will result in an additional burden to the pharmacy budget for anticoagulation therapy due to the increased drug costs. ${ }^{2}$ The current acquisition cost of a new anticoagulant drug is 2-3 euros/day versus 0.07 euros/day for warfarin. Such a policy for the entire Italian AF population (650,000 patients) would result in an incremental cost of 0.5 billion euros. The importance of an evidence based health policy has now been acknowledged worldwide, given the limited healthcare resources available. Reimbursement of new medicines is subjected to economic evaluation to improve efficient allocation of public resources. ${ }^{3,4}$

\section{Cost-effectiveness analysis}

Briefly, economic evaluation is a formal comparison of alternative actions in terms of costs and benefits. Cost-effectiveness analysis (CEA) is one of the four types of full economic evaluation (Table 1) in which costs are measured in monetary terms and outcomes are measured using a common unit of effect. ${ }^{3}$ This common unit is a clinical measurement when evaluating alternatives that produce the same effect (e.g. anti-hypertensive drugs and $\mathrm{mm} \mathrm{Hg}$ values reduction in blood pressure). If the alternatives do produce multiple effects, a more general measure is needed. This measure is the Quality Adjusted Life Year (QALY): CEA is also 
known as cost-utility analysis, when QALY is used to measure effectiveness. The QALY combines and weighs quantitative and qualitative data about life improvements, where 1 is the weight of full health and 0 is the weight of dead-equivalent..$^{3-5}$ Various methods for determining weights are available; of course, CEA including these methods are considered superior to others that do not. ${ }^{6,7}$

Cost-effectiveness analysis considers all the costs associated with a new technology/drug, not merely acquisition costs. Costs and savings accruing on the patients, their families and even the impact upon economic activities, have to be considered. Again, methods for these types of evaluation are well established. ${ }^{3}$ There are several different approaches to assess cost-effectiveness according to the data collection methods used (Table 2). CEA can be conducted alongside RCTs that provide evidence for efficacy and safety of a new technology/drug. This approach, often called piggy-back analysis, has several drawbacks that limit its application. These include: limited time-horizon, selected patient populations, economic data/analysis conducted for registration, not for pharmacoeconomic purposes. Economic modeling on the basis of the results of RCTs is perhaps the most accepted approach by decision makers to substantiate a reimbursement decision on a new drug; the decision tree, Markov and discrete event simulation are the most frequently applied. Clinical data from RCT are put together with information from epidemiological and cost-of-illness studies and other retrospective data sources, thus allowing a longer time horizon, and re-

Table 1. Classification of full economic evaluations according to the measurement of health gain.

\begin{tabular}{lccc}
\hline Type of evaluation & Measurement of health gain & Measurement of cost & Applicability \\
\hline Cost-minimization analysis & $\begin{array}{c}\text { Non-specified } \\
\text { (equal health gain) }\end{array}$ & Monetary value & $\begin{array}{c}\text { Comparison of medical } \\
\text { procedures with equal health gain }\end{array}$ \\
\hline Cost-effectiveness analysis & $\begin{array}{c}\text { Natural units (traditional } \\
\text { clinical trial end points) }\end{array}$ & Monetary value & $\begin{array}{c}\text { Comparison of medical } \\
\text { procedures with non-equal health gain } \\
\text { measurable in the same health dimension }\end{array}$ \\
\hline Cost-utility analysis & Quality-adjusted life-years & Monetary value & \begin{tabular}{c} 
Comparison of any medical procedures \\
\hline Cost-benefit analysis
\end{tabular} \\
Monetary value & Monetary value & $\begin{array}{c}\text { Comparison of any medical } \\
\text { and non-medical procedures and } \\
\text { investment options }\end{array}$ \\
\hline
\end{tabular}

Modified from Bodrogi et al., 2010. ${ }^{3}$

Table 2. Classification of economic evaluations according to the data collection method used.

\begin{tabular}{|c|c|c|}
\hline & Advantages & Disadvantages \\
\hline $\begin{array}{l}\text { Economic evaluation } \\
\text { alongside clinical trials }\end{array}$ & $\begin{array}{l}\text { Randomization (internal validity) } \\
\text { Low cost of economic data collection } \\
\text { Economic results are available before } \\
\text { reimbursement decisions }\end{array}$ & $\begin{array}{c}\text { Selected patient population } \\
\text { Protocol-induced costs } \\
\text { Limited time horizon } \\
\text { Monitoring of economic data is less strict } \\
\text { than of clinical variables } \\
\text { Calculation of statistical power is based } \\
\text { on efficacy end points } \\
\text { Economically meaningful events after clinical } \\
\text { end point and study drug discontinuation }\end{array}$ \\
\hline $\begin{array}{l}\text { Naturalistic } \\
\text { pharmacoeconomic studies }\end{array}$ & $\begin{array}{l}\text { Non-selected ordinary patients in routine care } \\
\text { settings (external validity) } \\
\text { Real world resource utilization and costs } \\
\text { independently of the study protocol } \\
\text { Easy monitoring if individual patient records in } \\
\text { payers' or managed care database can be linked } \\
\text { based upon individual patient ID } \\
\text { Large patient population }\end{array}$ & $\begin{array}{l}\text { Unpredictable data collection, complicated } \\
\text { study administration, lack of data monitoring } \\
\text { Selection bias (if no randomization) } \\
\text { Limited time horizon } \\
\text { Economic results available only after } \\
\text { reimbursement decisions }\end{array}$ \\
\hline
\end{tabular}

Economic modeling on the basis of prospectively collected clinical trial data
Economic modeling results available before major decisions (e.g. reimbursement)

Results can be generalized, adjusted to local medical practice and patient population
Results depend on appropriateness of modeling assumptions (e.g. model structure)

Known uncertainty in input parameters reduces the clarity of conclusions

Modified from Bodrogi et al., 2010.3 
sults that can be generalized and adjusted to local medical practice and patient population. The level of appropriateness of modeling assumptions (i.e. model structure, time horizon, sophistication of the model to differentiate clinically and economically meaningful outcomes) is of paramount importance in determining accuracy of CEA estimates. Input parameters are subject to considerable uncertainty both qualitatively and quantitatively, thus making conclusions less clear. ${ }^{3-5}$

Once the costs (money) and effects (QALY) of a new technology/drug have been established, they have to be paired with a comparator, usually the current gold standard in order to calculate the incremental cost effectiveness ratio (ICER). The ICER is calculated by dividing the difference in the expected costs by the difference in the expected QALY (Figure 1). This acquisition is very important from the perspective of a decision maker, because a threshold (i.e. maximum ICER value) could be established in order to accept/reject reimbursement for a new drug. ${ }^{3-5}$ The World Health Organization developed the CHOICE project (choosing interventions that are cost-effective) with the objective of providing policy makers with the evidence needed to allow them to decide on the interventions and programs which maximize health for the available resources. ${ }^{8}$ CHOICE uses gross domestic product (GDP) as a readily available indicator from which to derive the following three categories of costeffectiveness: i) highly cost-effective ( $<$ GDP per capita); ii) cost-effective (1-3 times GDP per capita); and iii) not cost-effective ( $>3$ times GDP per capita).

The National Institute of Health and Clinical Excellence (NICE) in the United Kingdom is perhaps the most famous regulatory agency dealing with decision making to accept or refuse to make new drugs available on the market based on CEA. When a NICE technology appraisal recommends use of a drug, treatment or other technology, the UK National Health Service must usually provide funding and resources for it within three months of the guidance being published.

NICE uses a standard approach to calculate cost and benefits: medicines whose ICER fall below the range of $£ 20,000-30,000$ per QALY gained are more likely to be approved than medicines whose ICER exceeds this national threshold. Nevertheless, the decisions of NICE are not wholly related to this unique parameter. Special circumstances if applicable, are considered and medicines with threshold-exceeding ICER are sometimes approved. ${ }^{9-11}$ Such special circumstances could include severity of underlying illness, end-of-life treatments, stakeholder pressure, significant innovation, disadvantaged population, or children.

\section{New anticoagulant drugs: economic evaluation}

Emerging health-economic evidence is available in the literature about new anticoagulant drugs. Most of this evidence is related to dabigatran, the first drug to generate evidence and to be introduced on the market.

\section{Dabigatran}

The most extensive and accurate CEA for dabigatran versus warfarin in AF has been produced by 2012 NICE technology appraisal document, on the basis of RELY trial results. ${ }^{12}$ The committee concluded that the most plausible ICERs for the whole population eligible for dabigatran were within the range normally considered a cost-effective use of National Health Service resources, i.e. less than $£ 20,000$ per QALY gained. Apart from this, some comments must be made about a number of controversial issues.

\section{C new - C old money \\ ICER $=\overline{\text { B new }- \text { B old }}=\frac{}{\text { QALY }}=$ money per QALY}

Highly cost effective

$\begin{array}{ll}\text { WHO } & <\text { GDP per capita } \\ \text { UK } & <\mathrm{f} 30,000\end{array}$

Cost effective

1-3 GDP per capita

£30-60,000
Not cost effective

>3 GDP per capita

$>£ 60,000$

C, cost; B, benefit; GDP, gross domestic product; ICER, incremental cost effectiveness ratio; QALY, quality adjusted life year.

Figure 1. Incremental cost effectiveness ratio (ICER) calculation and ICER thresholds. 
1. Effectiveness of dabigatran compared with warfarin according to international normalized ratio (INR) control on the evidence that people with good INR control with warfarin may not gain additional clinical benefit by taking dabigatran. The clinical specialists emphasised the importance of the significantly lower rates of intracranial haemorrhage and haemorrhagic stroke associated with both doses of dabigatran compared with warfarin in the RE-LY trial, and that this effect is maintained in people with good INR control. The Committee heard that haemorrhagic stroke and intracranial haemorrhage have devastating and life-threatening consequences and concluded that the lower rates associated with dabigatran represent an important advance in the treatment of atrial fibrillation alongside reduction in ischaemic stroke. $^{12}$

2. Restriction of dabigatran to patients with poor INR control. A time on therapeutic range (TTR) of more than $75 \%$ was calculated for warfarin in order to obtain an ICER for dabigatran above the threshold of $£ 30,000$ per QALY. This is well above the TTR of RELY (72\%) and of UK practice (67\%), and perhaps of the general real world, given the high standards of the UK anticoagulation service. The Committee was satisfied that the technology was a cost-effective treatment for the whole patient group. It noted that robust evidence of differential clinical effectiveness and cost effectiveness, with clear justification of the threshold level chosen, would be needed to select out a subgroup, based on INR control, for whom dabigatran would not be recommended. ${ }^{12}$

Dabigatran CEA in AF have been conducted in other countries by local health services. Three studies demonstrated the cost-effectiveness of abigatran across the USA. Freeman and Homan yielded an ICER less than 50,000 US dollars (USA ICER threshold) in the whole AF population and in patients with previous stroke/transient ischemic attack, respectively, while Shah documented a cost effectiveness only in people at high risk of hemorrhage or high risk of stroke unless international normalized ratio control with warfarin was excellent. From the perspective of Canadian Health Services, dabigatran versus warfarin is cost-effective with an ICER of 10,440 Can dollars/QALY.

Cost-effectiveness analyses have also been conducted across the European Union. According to national health services, dabigatran is considered cost-effective versus warfarin in AF patients in Belgium, Denmark, Sweden, UK and Spain. A recent paper extends the same results to Switzerland ${ }^{13-23}$ (Table 3).

\section{Rivaroxaban}

Evidence from the ROCKET trial was the basis for a NICE technology appraisal document on rivaroxaban..$^{24}$ The most plausible ICER for rivaroxaban versus warfarin lies between $£ 2870$ and $£ 29,500$ per QALY gained, given that the principle driver of CEA and the main source of uncertainty was the cost of anticoagulation monitoring. However, this ICER value is not above the threshold of $£ 30,000$ and rivaroxaban is considered a cost-effective option in AF patient management in the UK.

No clinically relevant subgroups for which there is evidence of differential effectiveness were identified. Rivaroxaban is a cost-effective treatment for the whole population of patients with AF, independently of risk stratification (CHADS2 score) and poor/good INR control (TTR).

Rivaroxaban has been evaluated for cost-effective-

Table 3. Dabigatran: cost-effectivess analysis data.

\begin{tabular}{|c|c|c|c|c|}
\hline Study & Country & Treatment & Year of costing & ICER/QALY gained \\
\hline \multicolumn{5}{|l|}{ Markov model } \\
\hline Freeman $^{13}$ & USA & Dabigatran $150 \mathrm{mg}$ & 2008 & \$US 45.372 \\
\hline Kamel $^{14}$ & USA & Dabigatran $150 \mathrm{mg}$ & 2010 & \$US 25.000 \\
\hline Kansal $^{15}$ & UK & Dabigatran $150 \mathrm{mg}$ & 2010 & $£ 4.831$ \\
\hline Langkilde $^{16}$ & Denmark & Dabigatran $150 \mathrm{mg}$ & 2011 & $€ 6.950$ \\
\hline $\mathrm{NICE}^{12}$ & UK & Dabigatran $150 \mathrm{mg}$ & 2011 & $£ 6.264$ \\
\hline Sha and Gage ${ }^{17}$ & USA & Dabigatran $150 \mathrm{mg}$ & 2010 & \$US 86.000 \\
\hline Sorensen ${ }^{18}$ & Canada & Dabigatran $150 \mathrm{mg}$ & 2010 & $\$$ Can 10.440 \\
\hline Pletscher ${ }^{19}$ & Switzerland & Dabigatran $150 \mathrm{mg}$ & 2011 & CHF10.215 \\
\hline Wouters ${ }^{20}$ & Belgium & Dabigatran $150 \mathrm{mg}$ & 2011 & $€ 5296$ \\
\hline Gonzales-Juanatey $^{21}$ & Spain & Dabigatran $150 \mathrm{mg}$ & 2010 & $€ 17.581$ \\
\hline Davidson $^{22}$ & Sweden & Dabigatran $150 \mathrm{mg}$ & 2011 & $€ 12.449$ \\
\hline $\begin{array}{l}\text { Discrete-event-simulation } \\
\text { Pink }^{23}\end{array}$ & UK & Dabigatran $150 \mathrm{mg}$ & 2009 & $£ 23.082$ \\
\hline
\end{tabular}

ICER, incremental cost effectiveness ratio; QALY, Quality Adjusted Life Year 
ness from the perspective of the US health services. The ICER for rivaroxaban was $\$ 27,498$ per QALY, well below the US-ICER threshold of $\$ 50,000$ per QALY. Again, the major driver of CEA was the lower hazard of intracranial hemorrhage with rivaroxaban..$^{25}$

\section{Apixaban}

Very recently, NICE evaluated apixaban for costeffectiveness on the data from the ARISTOTLE randomized clinical trial. ${ }^{26}$ The same standard approach used for dabigatran and rivaroxaban appraisal was used. The Committee concluded that apixaban had been shown to be cost-effective compared with warfarin, the most plausible ICER being less than $£ 20,000$ per QALY gained. Again, apixaban is a recommended option for the whole AF population, independently of CHADS2 score and TTR.

A comment was made about the different calculated ICERs for new anticoagulants. The ICER for apixaban was lower than that for dabigatran that was in turn lower than that for rivaroxaban, giving rise to the possibility of a different cost effectiveness among the drugs. There is, however, considerable uncertainty about the relative treatment effects of the drugs, arising from the base-line characteristics of people included in the trials and no firm conclusion can really be drawn on this issue. The Committee concluded that, for the moment, there was insufficient evidence to distinguish between the cost effectiveness of apixaban, dabigatran and rivaroxaban.

\section{Conclusions}

Economic evaluation and CEA conducted in European and North American countries have demonstrated the cost effectiveness of dabigatran, rivaroxaban and apixaban compared to warfarin for stroke prevention, in atrial fibrillation. In other words, the expected benefits, progressively increasing over time, far outweigh the higher initial acquisition cost. From the perspective of a regulatory agency, responsible for reimbursement of the cost of the drug, the number of patients subjected to treatment is of paramount importance for cost estimation. While no restriction to subgroup is applied to the population of patients with AF in Europe, Canada or the US, in Italy, the national local regulatory agency (Agenzia Italiana del Farmaco, AIFA) has still not made such a decision.

\section{References}

1. Masotti L, Di Napoli M, Ageno W, et al. Direct oral anticoagulants for secondary prevention in patients with nonvalvular atrial fibrillation. Ital J Med 2013;7(s8):8-21.
2. Atay JK, Fiumara K, Piazza G, et al. Hospital budget implications of substituting dabigatran for warfarin in an anticoagulation service. Clin Appl Thromb Hemost 2012;18:181-4.

3. Bodrogi J, Kalo Z. Principles of pharmacoeconomics and their impact on strategic imperatives of pharmaceutical research and development. Br J Pharmacol 2010; 159:1367-73.

4. Drummond MF, Sculpher MJ, Torrance GW, et al. Methods for the economic evaluation of health care programmes, 3rd ed. Oxford: Oxford University Press, 2005.

5. Edlin R, Round J, Hulme C, McCabe C. Cost effectiveness analysis and efficient use of the pharmaceutical budget: the key role of clinical pharmacologists. $\mathrm{Br} \mathrm{J}$ Clin Pharmacol 2010;70:350-5.

6. Brazier JE, Ratcliffe J, Tsuchiya A, Salomon J. Measuring and valuing health benefits for economic evaluation. Oxford: Oxford University Press; 2006.

7. Arnold D, Girling A, Stevens A, Liliford R. Comparison of direct and indirect methods of estimating health state utilities for resource allocation: review and empirical analysis. Br Med J 2009;339:b2688.

8. World Health Organization. WHO cost-effectiveness thresholds. 2012. Available from: http:// www.who.int/ choice/costs/CER thresholds/en/index.html

9. McCabe C, Claxton K, Culyer AJ. The NICE cost-effectiveness threshold: what it is and what that means. Pharmaco Economics 2008;26:733-44.

10. Culyer AJ, McCabe C, Briggs AH, et al. Searching for a threshold, not setting one: the role of the National Institute for Health and Clinical Excellence. J Health Serv Res Policy 2007;12:56-28.

11. Rawlins M. Pharmacoeconomics: NICE's approach to decision-making. $\mathrm{Br} \mathrm{J}$ Clin Pharmacol 2010;70: 346-9.

12. NICE Technology Appraisal Guidance 249 Dabigatran etexilate for the prevention of stroke and systemic embolism in atrial Fibrillation. Issued: March 2012. Available from: http://www.nice.org.uk/nicemedia/live/ 13677/58470/58470.pdf

13. Freeman JV, Zhu RP, Owens DK, et al. Cost-effectiveness of dabigatran compared with warfarin for stroke prevention in atrial fibrillation. Ann Intern Med 2010; 154:1-11.

14. Kamel H, Johnston SC, Easton JD, Kim AS. Cost-effectiveness of dabigatran compared with warfarin for stroke prevention in patients with atrial fibrillation and prior stroke or transient ischemic attack. Stroke 2012; 43:881-3.

15. Kansal AR, Sorensen SV, Gani R, et al. Cost-effectiveness of dabigatran etexilate for the prevention of stroke and systemic embolism in UK patients with atrial fibrillation. Heart 2012;98:573-8.

16. Langkilde LK, Bergholdt Asmussen M, Overgaard M. Cost-effectiveness of dabigatran etexilate for stroke prevention in non-valvular atrial fibrillation. Applying RELY to clinical practice in Denmark. J Med Econ 2012; 15:695-703.

17. Shah SV, Gage BF. Cost-effectiveness of dabigatran for stroke prophylaxis in atrial fibrillation / clinical perspective. Circulation 2011;123:2562-70.

18. Sorensen SV, Kansal AR, Connolly S, et al. Cost-effec- 
tiveness of dabigatran etexilate for the pre-vention of stroke and systemic embolism in atrial fibrillation: A Canadian payer perspective. Thromb Haemostasis 2011; 105:908-19.

19. Pletscher M, Plessow R, Eichler K, Wieser S. Cost-effectiveness of dabigatran for stroke prevention in atrial fibrillation in Switzerland. Swiss Med Wkly 2013; 143:w13732.

20. Wouters H, Thijs V, Annemans L. Cost-effectiveness of dabigatran etexilate in the prevention of stroke and systemic embolism in patients with atrial fibrillation in Belgium. J Med Econ 2013;16:407-14.

21. González-Juanatey JR, Álvarez-Sabin J, Lobos JM, et al. Cost-effectiveness of dabigatran for stroke prevention in non-valvular atrial fibrillation in Spain. Rev Esp Cardiol 2012;65:901-10.

22. Davidson T, Husberg M, Janzon M, et al. Cost-effectiveness of dabigatran compared with warfarin for patients with atrial fibrillation in Sweden. Eur Heart J 2013; 34:177-83.

23. Pink J, Lane S, Pirmohamed M, Hughes DA. Dabigatran etexilate versus warfarin in management of non-valvular atrial fibrillation in UK context: Quantitative benefitharm and economic analyses. BMJ 2011;343.

24. NICE Technology Appraisal Guidance 256. Rivaroxaban for the prevention of stroke and systemic embolism in people with atrial fibrillation Issued: May 2012. Available from: http://www.nice.org.uk/nicemedia/live/ 13746/59295/59295.pdf

25. Lee S, Anglade MW, Pham D, et al. Cost-effectiveness of rivaroxaban compared to warfarin for stroke prevention in atrial fibrillation. Am J Cardiol 2012;110:845-51.

26. NICE Technology Appraisal Guidance 275. Apixaban for preventing stroke and embolism in people with Atrial Fibrillation. February 2013. Available from: http://www. nice.org.uk/nicemedia/live/14086/62875/62875.pdf 